\title{
Adopting MQTT for a multi protocols IoMT system
}

\author{
Bilal Asaad Mubdir'1, Hassan Mohammed Ali Bayram² \\ ${ }^{1}$ Department of Communication Engineering, Sulaimani Polytechnic University, Sulaymaniyah, Iraq \\ ${ }^{2}$ Department of Computer Engineering, Koya University, Erbil, Iraq
}

\begin{abstract}
Article Info
Article history:

Received Jan 14, 2021

Revised Jul 23, 2021

Accepted Aug 11, 2021

\section{Keywords:}

IoMT

IoT

Microcontroller

MQTT

Raspberry Pi

WSN

ABSTRACT

Coronavirus disease (COVID-19) altered the way of caregiving and the new pandemic forced the health systems to adopt new treatment protocols in which remote follow-up is essential. This paper introduces a proposed system to link a remote healthcare unit as it is inside the hospital. Two different network protocols; a global system for mobile communication (GSM) and Wi-Fi were used to simulate the heath data transfer from the two different geographical locations, using Raspberry Pi development board and Microcontroller units. Message queuing telemetry transport (MQTT) protocol was employed to transfer the measured data from the healthcare unit to the hospital's Gateway. The gateway is used to route the aggregated health data from healthcare units to the hospital server, doctors' dashboards, and the further processing. The system was successfully implemented and tested, where the experimental tests show that the remote healthcare units using a GSM network consumed about $900 \mathrm{mWh}$. A high percentage of success data packets transfer was recorded within the network framework as it reaches $99.89 \%$ with an average round trip time (RTT) of 7.5 milliseconds and a data transfer rate up to $12.3 \mathrm{kbps}$.
\end{abstract}

This is an open access article under the CC BY-SA license.

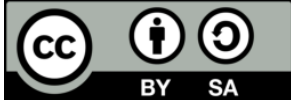

\section{Corresponding Author:}

Bilal Asaad Mubdir

Department of Communication Engineering, Sulaimani Polytechnic University

Sulaymaniyah, Iraq

Email: bilal.mubdir@spu.edu.iq

\section{INTRODUCTION}

Humans' health and from the earliest signs of history recording, thousands of years ago were of great interest since it is directly related to the continuity of human being and their civilizations. Passing through several stages of development, healthcare witnessed a marvelous step ahead with the appearance of both embedded systems technology and the internet. Embedded systems and the internet were the basic elements of creating the new age namely the internet of things (IoT) age. IoT affects humans' lifestyles in every aspect. As an ecosystem, IoT invaded the industry, education institutes, scientific research centers, world economy, military, and healthcare industries. Internet of medical things (IoMT) with a market share of about $6 \%$ of the total IoT market, untangled healthcare for both caregivers and caretakers [1].

Modern healthcare systems including patient remote monitoring systems, online reservation systems, remote and endoscopic surgeries, and patients' history records and database, are all iomt applications and outcomes. A patient remote control system, as an example, enhanced the way Elderlies, diabetic patients, patients with heart diseases, and special needs patients are receiving the care they need with more suitable and comfortable ways for them and the caregivers. In late 2019, a new coronavirus (COVID-19) invaded the entire world and was declared as a pandemic by the World Health Organization (WHO) on March 2020 [2]. COVID-19 hit more than 40 million active cases within a period that is less than a year. The rapid and progressive increase in the number of confirmed cases pushed most of the health 
systems in many countries including developed countries to implement remote care procedures to deal with mild cases to decrease the load over the systems and preserve the valuable lives of the medical staff. Patients with a positive COVID-19 test and mild symptoms are remotely supervised by a healthcare giver to monitor their condition and treatment. An independent healthcare unit is assigned for each patient to measure essential vital signs and record the follow-up of the treatment.

The patient healthcare unit (PHU) is a sensor node in a wireless sensor network (WSN). The current pandemic (COVID-19) arises a special need of monitoring a patient remotely in a steady manner. Sensor nodes are usually linked using a networking protocol, i.e., Wi-Fi, long-range (LoRa), Bluetooth low energy (BLE), Zigbee within the coverage of the hospital. Any network protocol used in the WSN subjects the coverage to a specific limitation. The aforementioned patients-especially patients in rural areas- are in a region that is out of coverage of the hospital's WSN. Remotely located PHUs are to be linked with the hospital's server by a different type of networking protocol.

This paper discusses a proposed system in which two different nodes-within and out of-WSN coverage are integrated within a single WSN by the implementation of a global system for mobile communication (GSM) based IoT framework. The proposed system consists of three main parts: remote PHU, local PHU, and a gateway. The remote PHU is located out of the hospital's coverage, it is used to monitor the body temperature and the oxygen saturation (SPO2) in the patient's blood and programmed to send measured data to the gateway over the GSM network. On other hand, the same monitoring features are implemented in a local PHU that is located within the hospital and upload measured data to the gateway over a Wi-Fi network. The gateway was developed using open-source tools to facilitate the aggregated health data from all connected local and remote PHUs. Message queuing telemetry transport (MQTT) protocol is utilized to transfer the health data from each PHU to the gateway directly.

\section{INTERNET OF THINGS}

A new technological era (the IoT age) that have been vastly deployed connecting hundreds of millions of smart objects such as: machines, sensors, gadgets, and appliances. IoT systems make use of information aggregated by sensors via divergent communication technologies where all devices are linked in a network [3]. Latest technologies and enormous development in information gathering, networking, communication, sensors' manufacturing, cloud computing, artificial intelligence, software, and algorithms helped the domination of IoT systems over every process and daily activities [4]. Home monitoring in 1970 ignites the revolution of developing and implementing IoT systems [5].

A significant role is guided information gathering and decision making into a glorious level which is highlighted by figures of IoT-based devices will be in tens of billions by 2022 [6]. As predicted by Cisco in their annual internet report over the period between 2018 and 2023, IoT-connected devices shall share 50\% of the entire connections in 2023. Cisco's whitepaper highlighted that out of 14.7 billion IoT-connected devices, IoMT and smart health systems shall hit 735 million connected devices by 2023 which is about $5.6 \%$ of the machine to machine (M2M) connection estimated as shown in Figure 1. Hence, IoT-based healthcare systems form $2 \%$ of the total number of internet-connected devices [1].

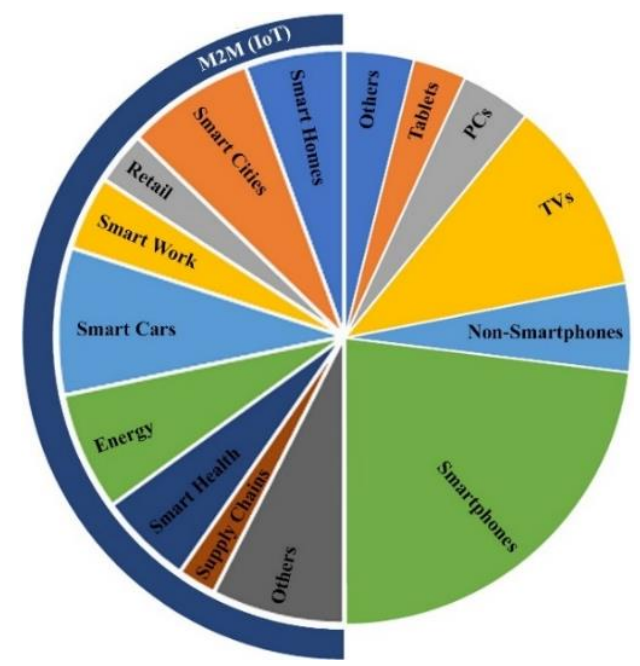

Figure 1. Expected M2M internet-connected devices in 2023 
The importance of IoT reclines in the presence of its systems in spacious types of applications where different categories of IoT systems have been used in industry, economic systems, monitoring, and security systems [5]. IoT systems fall into three categories: Monitoring and controlling, big data and business analytic, and information sharing and collaboration [7]. A major field of IoT is in the medical field where the term internet of medical things (IoMT) is used [8]. Embedding IoT technologies within healthcare systems bridle humans' errors and reduces expenditure figures [9]. IoMT pervades the medical field in countless applications such as; patient and elderly remote care systems, diseases diagnosing and treatment, remote surgical operations, and smart hospitals. Figure 2 shows the difference between the three and the five layers protocols' architecture of the IoT. An application layer of the five-layered architecture performs and replaces business, application, and service management layers in the three-layered architecture. The first and second layers in both architectures are the same with different names. Finally, the same set of protocols are used in both architectures. Figure 2 shows the three and five-layers architectures of IoT and the protocol used in each layer.

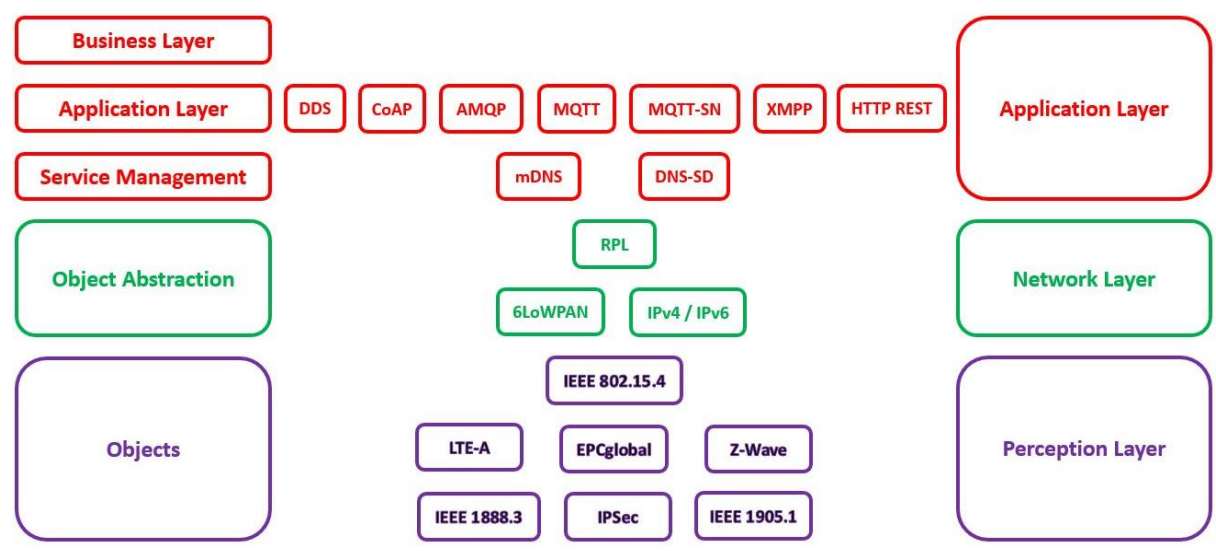

Figure 2. IoT layers' architecture and protocols

\section{INTERNET OF MEDICAL THINGS}

Layered architecture simplifies designing and implementing processes of a system, hence IoT and IoMT systems' implementation are based on a layered architecture [10], [11]. Three-layered, five-layered, middleware, and service-oriented architectures (SOA) are all assigned for the implementation of an IoT system [11]. Layered architecture organizes the levels where a system component is located. A physical layer is a spot where sensors and smart objects are installed to acquire the required data of a patient. A network layer; object abstraction layer is the playfield of the communication technologies such as 3G, BLE, Wi-Fi, and other technologies that are used to transmit the patient's biomedical signals throughout the system. Applications, user interface, service allocation, and the business model serve the integration of the system's beneficiaries by connecting service requests with service-providing parts of the system. Finally, data processing, storage, and artificial intelligence schemes are distributed among several layers to contribute an accurate and precise service to the use [10].

The internet of medical things and its applications infiltrate all aspects of health-giving systems via a variety of applications summed up under different health-related scenarios [9]. Heart diseases and heart status monitoring are found in [12], [13]. Furthermore, [14], [15] discussed the glucose levels and diabetic patient monitoring. neurological and brain diseases such as Alzheimer's disease in [16], dementia assessment in [17], and elderly monitoring system including fall detection system as in [18]. An ingestible device with an antenna and propagation to transmit images of the internal of the human body as a replacement of ordinary endoscopy [19]. Otoom and his colleagues proposed an IoT-based framework in [20], their proposed framework works on detection, monitoring, and data analysis for both suspected and identified cases of the new coronavirus (COVID-19). Digital technologies that have been employed for analyzing, detecting, monitoring, and fighting COVID-19 are discussed in [21]. Ting et al. [21] highlighted IoT as one of the most significant technologies leading the new era of medical care.

Despite the challenges of identification, sensing, and computing in an IoT system, communication is the utmost challenge facing IoT developers [10]. An increase in IoT systems deployed means an increase in connected smart devices and objects which results in an enormous amount of data to aggregate, process, and 
transmit [4]. Dealing with such an amount of data is eased by the use of the previously mentioned layered architecture. Each layer has its own set of protocols. The selection of the proper set of protocols depends on the type of the deployed system, type of aggregated data, network structure, and topology. CoAP, MQTT, hypertext transfer protocol (HTTP), data distribution service (DDS), and others are grouped as application layer protocols. Multicast domain name service (mDNS) and domain name service service discovery (DNSSD) are service delivery protocols. RPL, 6LowPAN, BLE, and LTE-A are the infrastructure protocols in addition to the influential protocols [4], [10].

\section{MQTT PROTOCOL}

The application layer is packed with dozens of protocols that serve the same cause of better system performance [4]. The constrained application protocol (CoAP) and the message queue telemetry transport protocol (MQTT) are at the top of the list of application protocols [22]. Both CoAP and MQTT are publisher/subscriber protocols with about the same features regarding power consumption and ease of implementation within a system [4], [10], [22]. MQTT outperform CoAP in term of system with lower bandwidth links, higher throughput and less latency [4], [22], [23].

MQTT lacks nodes' discovery but it features simplicity, ease of implementation, lightweight, minimum battery and memory requirement, less bandwidth in comparison to other protocols, the transporting overhead is small and the protocol exchange is minimized [4], [10], [22], [23]. The previously mentioned features of MQTT take the edge of network traffic off, hence considered as an excellent choice of IoT systems [22], [23]. Transport layer security (TLS) and secure sockets layer (SSL) protocols are employed by MQTT as it runs over transmission control protocol (TCP) to stand as the needed security levels [4], [10].

Asynchronous MQTT protocol outshines other protocols by its distinctive QoS with only 6 bytes used as its header and overhead [24]. Based on the client/server structure, and as any other publish/subscribe protocol, MQTT uses a broker (server) to collect packets of data from sensor nodes and serve subscribed clients upon requests [23]. MQTT server, also referred to as broker or HiveMQ, works as a mediator among connected clients. HiveMQ manages to publish, subscribe, unsubscribe, and disconnect processes among connected clients. Client-broker interaction is called a session where data (application message) is exchanged. An application message carries its QoS and topic (address).

MQTT is suitable for medical applications as it has been designed to be one-to-many and many-tomany which allows a client to subscribe to more than a topic at a time. The protocol is also space decoupled as the broker is the central node of the network and all exchanged data are going through, hence reducing the message overhead. The space decoupling eliminates the nodal dependency but makes the entire protocol broker dependent [23]-[25].

Time decoupling is another feature of the MQTT, it simply disconnects inactive clients and improves power consumption figures. The protocol uses a queue to save publishers' messages for subscribed but inactive clients. MQTT implements security procedures using different schemes and across several layers. security options include authentication, authorization, and data confidentiality.

An illustrated explanation of the MQTT quality of services is shown in Figure 3. At most one quality of service is illustrated in red where the temperature sensor is sending its readings to the broker without the need for any acknowledgment nor reply. QoS1 shown in blue highlights at least one quality of service. In this type, a patient is publishing his test result to the broker who is publishing it to the physician. Both physician and the broker are sending a reply at least once.

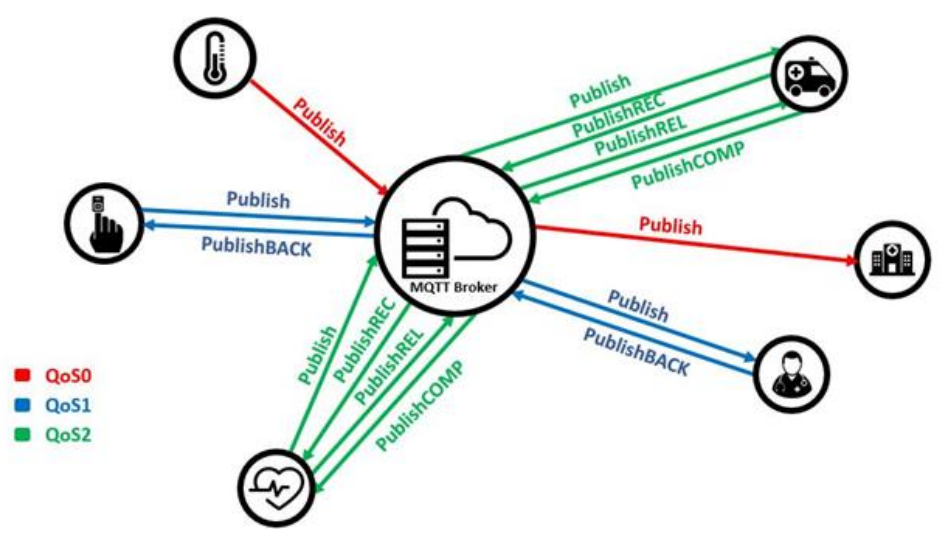

Figure 3. Publish/subscribe MQTT quality of service 
The last quality of service in the MQTT protocol is called exactly one and known as QoS2 or exactly once. The exactly once is considered the safest QoS with four parts handshake procedure between the sender and the receiver. The MQTT quality of services are listed below [24]:

- At most once (Level 0): an application message is sent one and no recipient acknowledgment is requested send and forget).

- At least once (Level 1): a publisher keeps sending the application message until at least once an acknowledgment is received from a subscriber.

- Exactly once (Level 3): a four-way handshaking system is implemented as the most reliable QoS of the MQTT protocol.

\section{PROPOSED SYSTEM DESIGN AND IMPLEMENTATION}

A study case of a hospital that has a simple WSN based WiFi network that is internet enabled, and several sensor nodes are linked together within the local area network (LAN) as shown in Figure 4. In Figure 4, data routed from the nodes toward the gateway that represents the edge node concerning any IoT system. Edge node is connected to the internet to aggregate and process the health data, then send it to the doctors over the internet cloud or for logging that data in the hospital itself. A new sensor node is intended to be linked to the network in a rural area as shown in Figure 4, where it is located $10 \mathrm{~km}$ far away from the hospital.

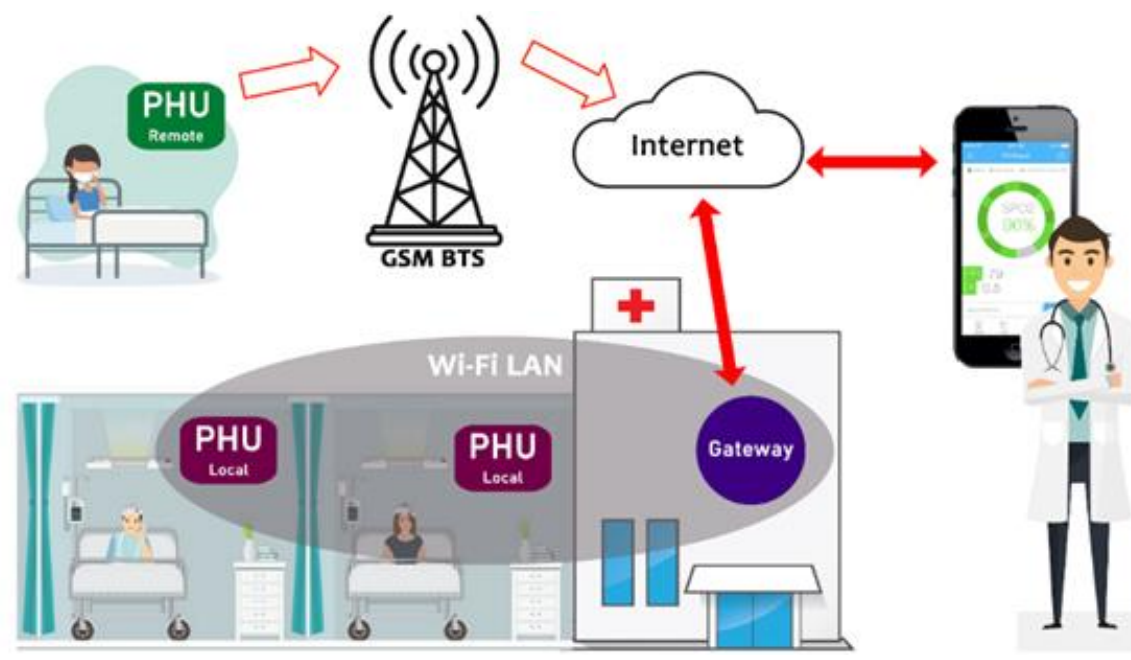

Figure 4. Simple WSN connected to the internet

Referring to the above case study, where there is no common network protocol between the remote and the local PHUs. Thus, MQTT based system was proposed to collect the data from the two different schemes of nodes, where the system comprises the two different communication network protocols, Wi-Fi and GSM. The data broadcasted from any PHU toward the gateway is exchanged based on MQTT protocol over GSM network for the remote PHU, and over Wi-Fi for the local PHU. Illustrating the implementation of the proposed system made easier by dividing it into three main parts, gateway, local PHU, and remote PHU.

\subsection{System gateway}

The edge of the fogy layer of any IoT system is simply the gateway. In the proposed system, a gateway was developed by utilizing an ARM-based development board operated by Linux OS (Raspberry Pi 3B) to receive the data transmitted from the nodes. The origin of Raspberry Pi is Cambridge's Computer Laboratory, where it was invented in 2007, but it was released in markets in 2012 by Raspberry Pi Foundation. Raspberry Pi board represents a small multipurpose microprocessor computer system with a lot of features and capabilities especially when it is operated under Linux OS. It provides an easy method to build custom servers or gateways with aid of fully controlled general-purpose input/output (GPIO) and the integrated Wi-Fi and Bluetooth transceivers [26], [27].

The gateway is configured to communicate with remote and local PHUs through port number 1883 to the MQTT broker. An open source MQTT broker (eclipse mosquitto) was installed under Linux and used 
by the proposed system [28]. The configuration of the gateway enables the broker to receive the messages from local PHUs over the local network using the local IP. On other hand, messages from remote PHU are received via the public IP of the gateway itself to the internet service. Furthermore, the messages are stored in different tables for further processing by another server, or any endpoint as shown in Figure 5.

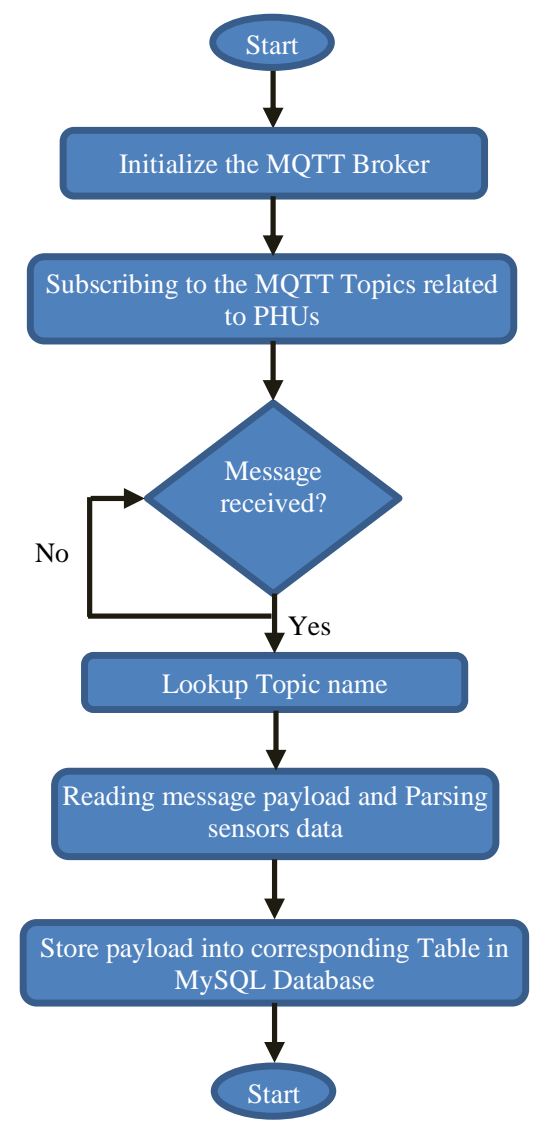

Figure 5. Python program flowchart of the gateway

A python script was developed to perform the processing of the received data, storing data into MySQL database, and display the received data from nodes in a webpage designed using cascading style sheets (CSS), PHP hypertext preprocessor (PHP), and hypertext markup language (HTML). To make it easier for the doctor to follow up the patients' status, curves are generated from the received data stores in the database based on standard fetching methods and opensource libraries from [29].

\subsection{Remote PHU}

Remote PHU was designed to communicate to the gateway over the GSM network as explained before. ATmega328 MCU has been used to implement the node. In addition to the default IO ports that are available in many MCU, ATmega328 has a built-in universal asynchronous receiver-transmitter (UART) circuit that enables the MCU to establish a connection with any Serial peripheral such as communication modules, sensors, and ther screens [30]. MAX30100, Pulse Oximeter designed for wearable health applications, has been used to measure the oxygen saturation in the patient's blood (SPO2) [31]. Besides SPO2, the body temperature has been measured by using the MLX90614 infrared thermometer that is specified for medical purposes [32], where the two sensors were interfaced to the MCU via the I2C protocol. Moreover, SIM800L GSM/GPRS module interfaced to the MCU over the Serial communication port to establish the connection to the GSM network as shown in the circuit diagram of the Figure 6.

TCP/UDP stack is embedded in the SIM800L module that is manufactured by SIMCOM [33], where it is a key requirement to communicate with servers and handle MQTT protocol and messages. By using $\mathrm{C} / \mathrm{C}++$ for the microcontroller and the AT commands of the SIM800L module, the remote PHU has been programmed to read the sensor and send its value to the gateway. 


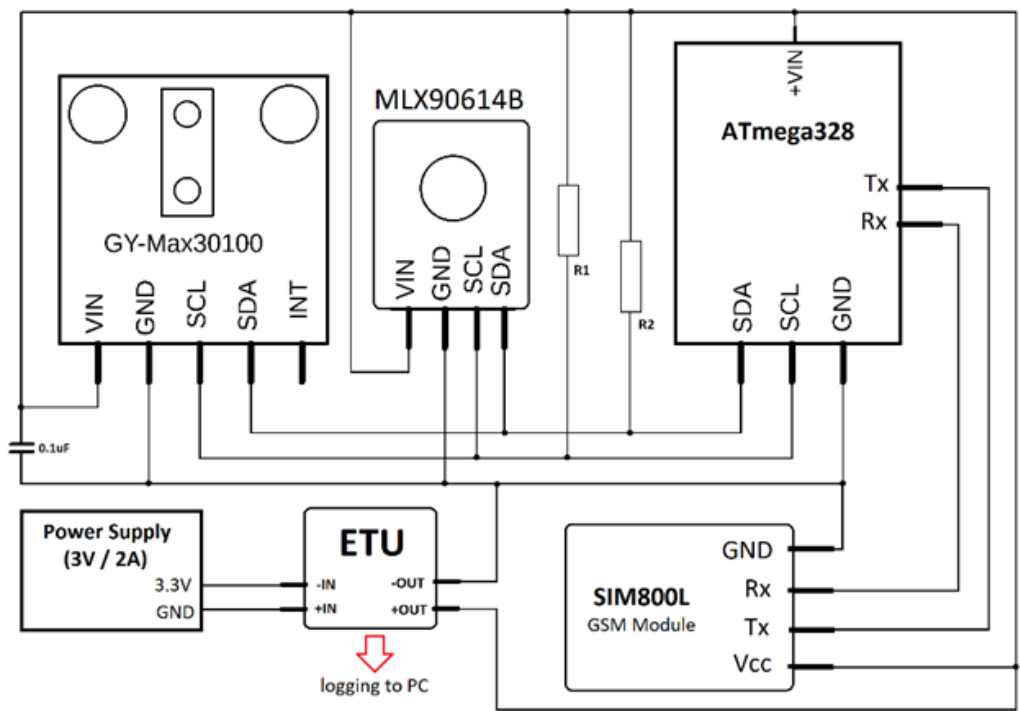

Figure 6. Circuit diagram of the Remote PHU

\subsection{Local PHU}

ESP8266 has been selected to implement the local PHU, where the same sensors (MAX30100, and MLX90614) were used also to build the prototype of the local PHU as shown in the circuit diagram of Figure 6. ESP8266 is an SoC with full MCU capabilities and Wi-Fi features supported by TCP/UDP protocol introduced by espressif systems [34]. The module is available in a development board called "ESP-12E", where it has been used to communicate with the gateway over the LAN.

$\mathrm{C} / \mathrm{C}+$ was also used to program the ESP8266 SoC, where the same procedure was applied. The values of the sensors are sent to the gateway over Wi-Fi based on the MQTT protocol. Both nodes were implemented with a custom energy testing unit (ETU). The ETU logs the energy measurements data to a PC for testing and verification purposes as shown in Figures 6 and 7.

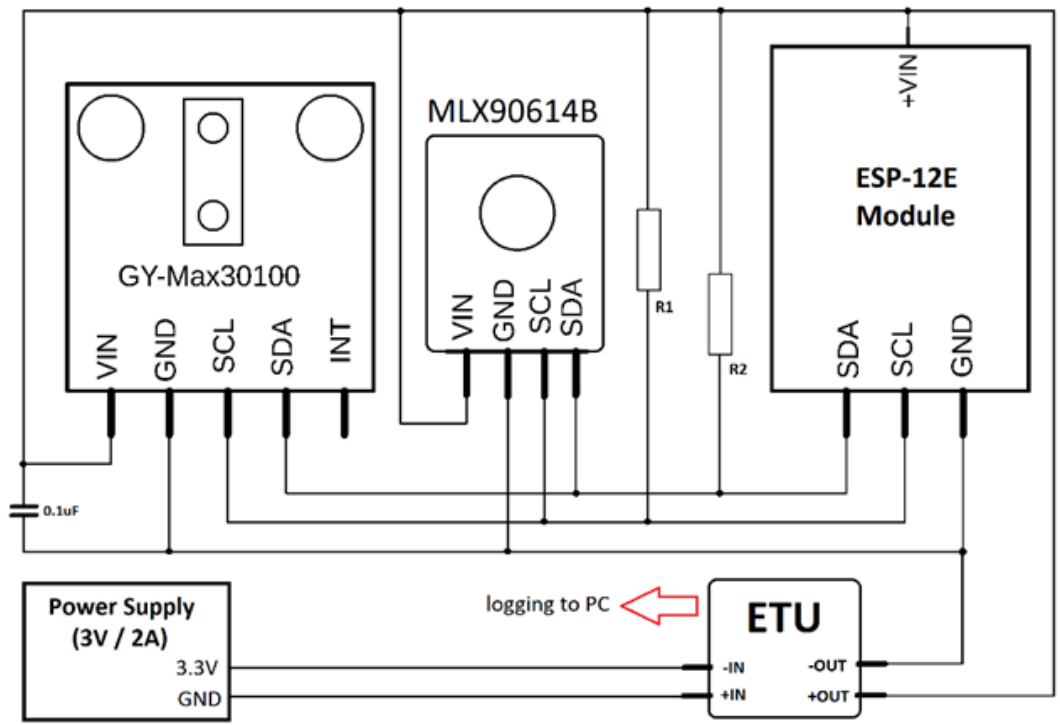

Figure 7. Circuit diagram of the typical local PHU

\section{SYSTEM EVALUATION AND RESULTS}

The proposed system has been tested after successful prototyping and implementation to prove the feasibility of the system and its compatibility. Two different types of tests were chosen to examine the 
system; the energy consumption test and network performance test. The remote PHU represents the core of this paper's problem statement, therefore both tests were applied only on the node for 60 minutes continuously. The two sensors reading, SPO2 and temperature are packed as payload in a total of 23 bytes with its header were transmitted frequently every 40 milliseconds during the whole 60 minutes in a single message with QoS equal to zero a setting for the MQTT.

\subsection{Energy test}

Sending a message over MQTT normally takes few milliseconds and consuming very little amount of energy around microwatts or less where it is very hard to be measured instantaneously. For that reason, the ETU was used to measure the average consumed energy every 1 millisecond by sensing the current and the voltage applied to the remote PHU and log accurate energy consumption. Finally, accumulated energy consumption data are recorded every 1 minute according to (1):

$$
E=\sum_{n=1}^{60000} \frac{V_{n} I_{n}}{6 \times 10^{4}}
$$

where;

$E \quad$ : accumulated energy for one minute

$V_{i} \quad$ : applied voltage on Remote PHU

$I_{i} \quad$ : applied current on Remote PHU

$n \quad$ : time slot of the measurement (1 millisecond)

The 60,000 measurements were collected and obtained from the ETU, where the average energy consumption per minute was about $15 \mathrm{mWh}$, and the total consumption was $900 \mathrm{mWh}$ for the one hour of the test.

\subsection{Networking test}

Connection speed and reliability between the remote PHU and the gateway is the most critical part to prove the system feasibility especially if the system collects critical data or if the data must be transmitted/received in real-time. One of the important metric parameters to test the connection health in a specific network is the round-trip time (RTT). The RTT test is performed usually by networkers and internet creators to diagnose the network's speed and reliability. Knowing that RTT represents the "total time it takes for the data to be sent plus the time it takes for the acknowledgment of that data to be received" [35]. RTT test was performed for all MQTT messages traveled from the Remote PHU to the gateway for 60 minutes as shown in Figure 8. Measuring the RTT could be made by capturing all messages broadcasted from the remote PHU to the gateway. For this purpose, a PC was used with an opensource application to capture the messages before going into the gateway by using a special network configuration shown in Figure 9.

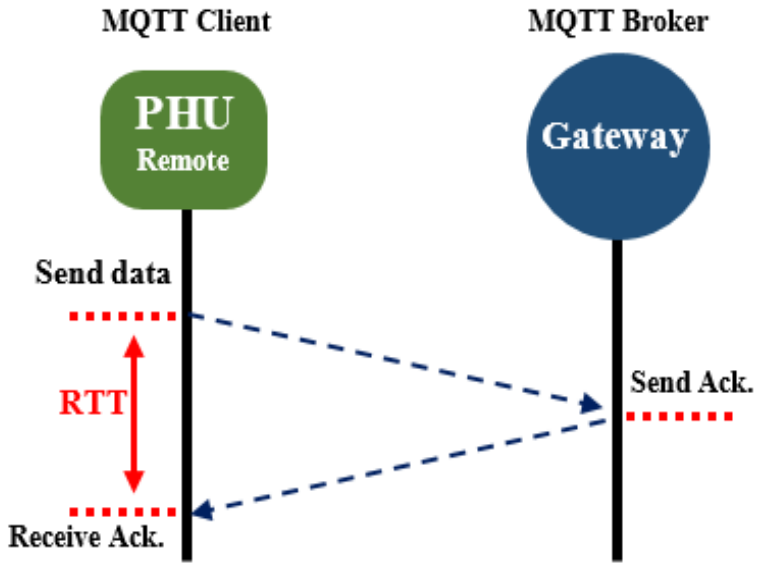

Figure 8. Round trip time (RTT) for the remote PHU

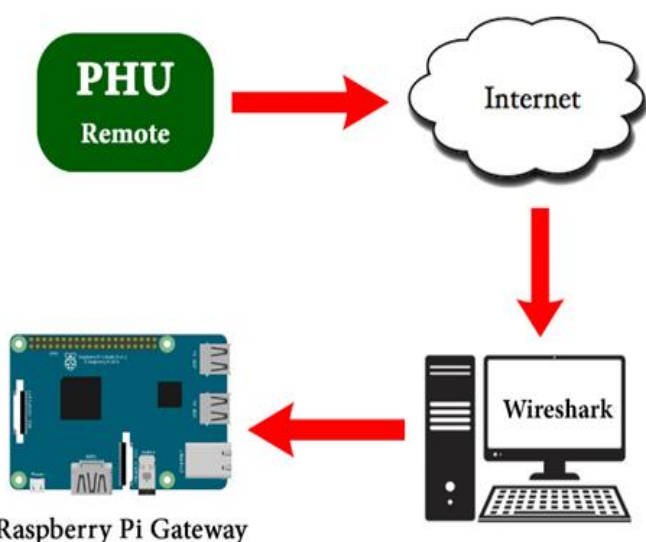

Figure 9. Network setting for capturing data

On PC and under Windows OS, Wireshark software was installed to capture and analyze the data. Wireshark is an open-source application that works as a sniffing tool within the network. It provides different tools and features for capturing, logging, filtering, and analyzing data packets that flow through the network and measure different parameters such as response time, and RTT [36]. 
After a continuous 60 minutes test, a total of 128,848 packets were transferred between the remote PHU and the gateway in both directions. Those packets represent the data packets sent with a total of 64,424 packets in addition to the same number of packets that represent the TCP acknowledgment packet from the gateway to the remote PHU. Errors, warnings, and notes captured by Wireshark for the whole test prove that there were about 76 missed or malformed packets out of 64,424 packets which result in only $0.11 \%$ of error during the test. Also, the I/O results show that the data transfer speed exceeds 1200 packets per minute or $12.32 \mathrm{kbps}$ as shown in Figure 10.

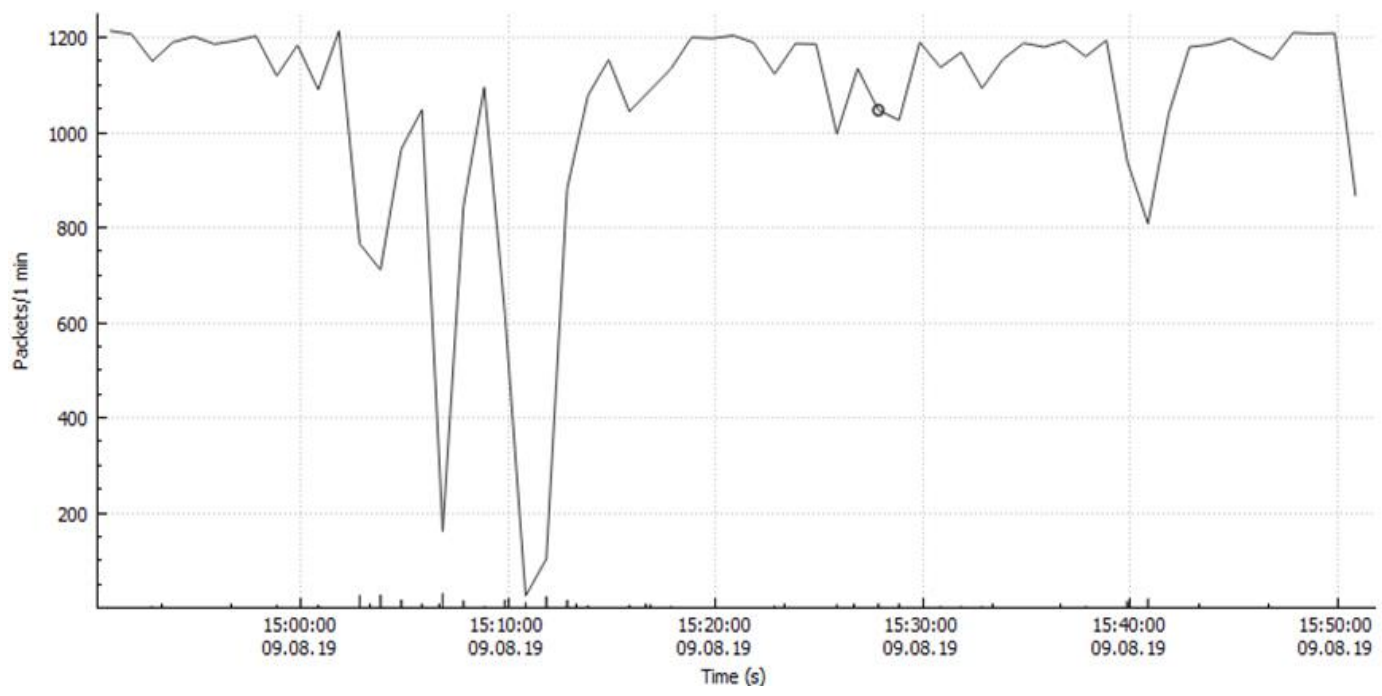

Figure 10. Packet transfer speed graph for captures packets

The results provide a clear image for the RTT pattern of the test by using the TCP steam graph that includes the RTT of every captured packet as shown in Figure 11, where it is seen the errors and resetting of the connections. Out of the 60 minutes test, a precise investigation show in Figure 12 was made on the time duration 440-460 seconds in Figure 11 to estimate the average RTT value. The RTT observed within this range is ranged from 12 milliseconds in its worst cases, to 2.5 milliseconds in the best cases and 7.25 milliseconds on average.

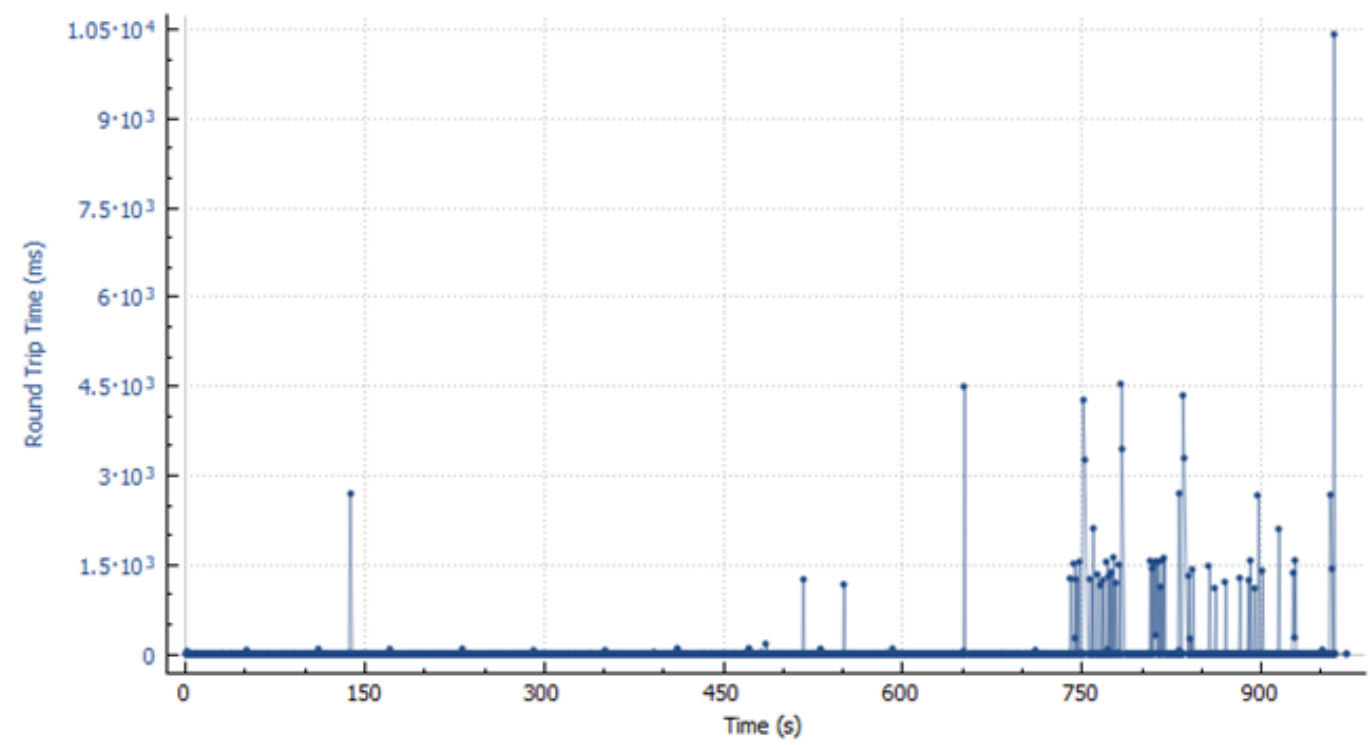

Figure 11. RTT for all captured packets within the test time 


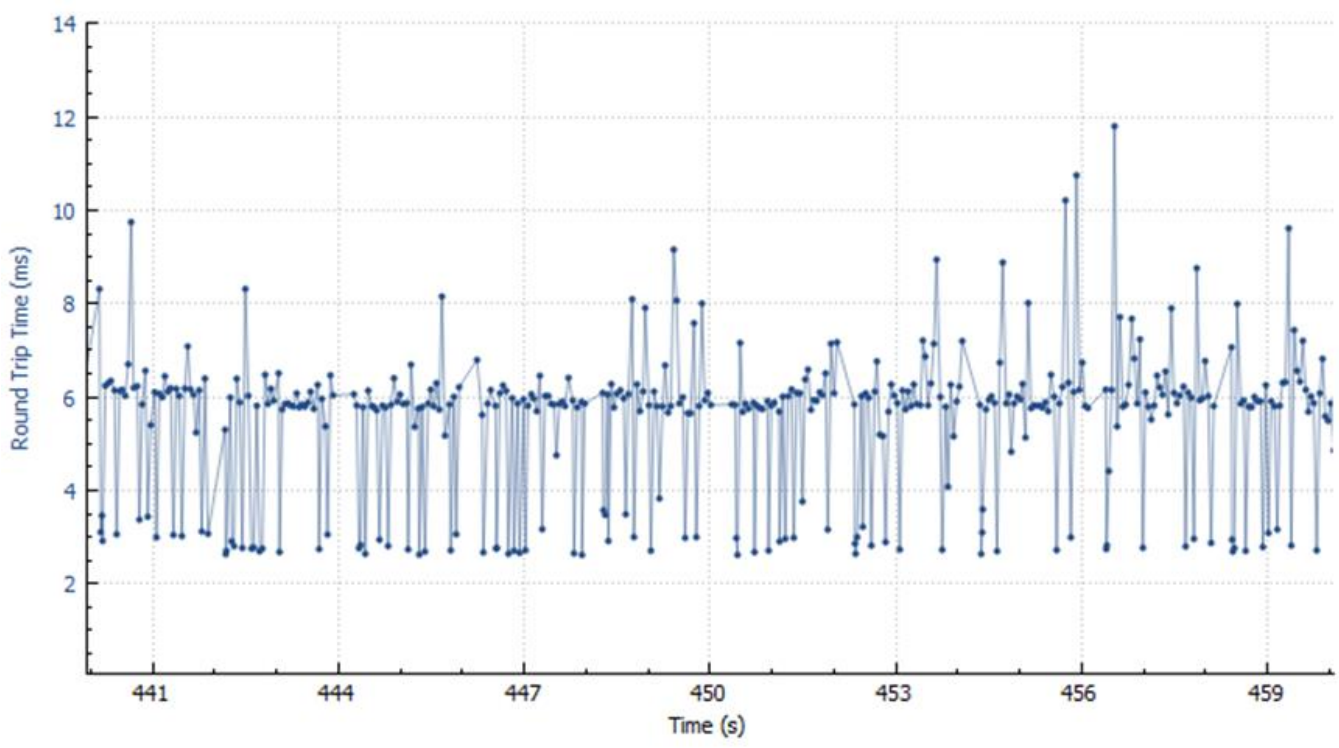

Figure 12. Precise look at the RTT during 440-460 seconds

\section{CONCLUSION}

Employing such a system for monitoring the health status of a patient remotely saves a lot of souls and efforts. The proposed system was successfully implemented and verified. Based on IoT principles, fully functional sensor nodes called PHU were developed to monitor the SPO2 and the body temperature of a patient located in a rural area that is out of the hospital's local network coverage. The proposed system uses the MQTT protocol due to its advantages in exchange the data in light packets even over low internet bandwidth. The remote PHU is linked to the hospital's WSN via the GSM network and the internet, whereas the local PHUs in the hospital is connected to the gateway over Wi-Fi. The test's results show that the proposed system is working perfectly, with total energy consumption of $900 \mathrm{mWh}$ for the remote PHU and 7.5 milliseconds as an average RTT for the health data transferred from the remote PHU to the gateway with a speed of up to $12.3 \mathrm{kbps}$ and total packet error of not more than $0.11 \%$.

\section{ACKNOWLEDGEMENTS}

All experimental tests and the hardware implementation were carried out at the Training and Development department, UrukTech Electronics Company, Iraq.

\section{REFERENCES}

[1] Cisco, "Cisco annual internet report (2018-2023) white paper," Cisco, 2020, [online] Available: https://www.cisco.com/c/en/us/solutions/collateral/executive-perspectives/annual-internet-report/white-paper-c11-741490.html (Accessed Aug. 31, 2021)

[2] World Health Organization, “Coronavirus disease 2019 (COVID-19)," Situation Report-51, 2019. [Online]. Available: https://www.who.int/docs/default-source/coronaviruse/situation-reports/20200311-sitrep-51-covid-19.pdf?sfvrsn=1ba62e57_10. (Accessed on August 31, 2021)

[3] J. Holdowsky, M. Mahto, M. E. Raynor, and M. Cooteeler, "Inside the internet of things (IoT) - A primer on the technologies building the IoT," Deloitte University Press, London, 2015.

[4] P. Sethi and S. R. Sarangi, "Internet of things: Architectures, protocols, and applications," Journal of Electrical and Computer Engineering, vol. 2017, pp. 1-25, 2017, Art. no. 9324035, doi: 10.1155/2017/9324035.

[5] K. Rose, S. Eldridge, and L. Chapin, "The internet of things: An overview understanding the issues and challenges of a more connected world," The Internet Society (ISOC), 2015

[6] A. S. El-Wakeel, J. Li, A. Noureldin, H. S. Hassanein and N. Zorba, "Towards a practical crowdsensing system for road surface conditions monitoring," IEEE Internet of Things Journal, vol. 5, no. 6, pp. 4672-4685, 2018, doi: 10.1109/JIOT.2018.2807408.

[7] I. Lee and K. Lee, "The internet of things (IoT): Applications, investments, and challenges for enterprises," Business Horizons, vol. 58, no. 4, pp. 431-440, 2015, doi: 10.1016/j.bushor.2015.03.008.

[8] S. K. Kharroub, K. Abualsaud, and M. Guizani, "Medical IoT: A comprehensive survey of different encryption and security techniques," 2020 International Wireless Communications and Mobile Computing (IWCMC), Limassol, Cyprus, 2020, pp. 1891-1896, doi: 10.1109/IWCMC48107.2020.9148287.

[9] S. Vishnu, S. R. J. Ramson, and R. Jegan, "Internet of medical things (IoMT)-An overview," 2020 5th International Conference on Devices, Circuits and Systems (ICDCS), Coimbatore, India, 2020, pp. 101-104, doi: 10.1109/ICDCS48716.2020.243558. 
[10] A. Al-Fuqaha, M. Guizani, M. Mohammadi, M. Aledhari, and M. Ayyash, "Internet of Things: A survey on enabling technologies, protocols, and applications," IEEE Communications Surveys \& Tutorials, vol. 17, no. 4, pp. 2347-2376, 2015, doi: 10.1109/COMST.2015.2444095

[11] M. Irfan and N. Ahmad, "Internet of medical things: Architectural model, motivational factors and impediments," 2018 15th Learning and Technology Conference (L\&T), 2018, pp. 6-13, doi: 10.1109/LT.2018.8368495.

[12] L. P. Malasinghe, N. Ramzan, and K. Dahal, "Remote patient monitoring: a comprehensive study," Journal of Ambient Intelligence and Humanized Computing, vol. 10, pp. 57-76, 2019, doi: 10.1007/s12652-017-0598-x.

[13] T. Szydło and M. Konieczny, "Mobile devices in the open and universal system for Remote patient monitoring," IFACPapersOnLine, vol. 48, no. 4, pp. 296-301, 2015, doi: 10.1016/j.ifacol.2015.07.050.

[14] M. Ali, L. Albasha, and H. Al-Nashash, "A Bluetooth low energy implantable glucose monitoring system," 2011 8th European Radar Conference, Manchester, 2011, pp. 377-380.

[15] K. A. U. Menon, D. Hemachandran and T. K. Abhishek, "A survey on non-invasive blood glucose monitoring using NIR," 2013 International Conference on Communication and Signal Processing, Melmaruvathur, 2013, pp. 1069-1072, doi: 10.1109/iccsp.2013.6577220.

[16] K. Y. Lam, N. W. H. Tsang, S. Han, W. Zhang, J. K. Y. Ng, and A. Nath, "Activity tracking and monitoring of patients with alzheimers disease," Multimedia Tools and Applications, vol. 76, no. 1, pp. 489-521, 2017, doi: 10.1007/s11042-015-3047-x.

[17] A. Mohamed, P. Novais, A. Pereira, G. V. González, and A. F. Caballero, "Ambient intelligence - software and applications," 1st ed. Springer International Publishing, 2015, doi: 10.1007/978-3-030-24097-4.

[18] S. Solórzano, M. R. Ortiz, R. L. Molina, J. Clairand, and D. P. Espín, "Home tele-assistance system for elderly or disabled people in rural areas," 2018 International Conference on Democracy \& eGovernment (ICEDEG), Ambato, 2018, pp. 380-385, doi: 10.1109/ICEDEG.2018.8372306.

[19] M. R. Yuce and T. Dissanayake, "Easy-to-swallow antenna and propagation," IEEE Microwave Magazine, vol. 14, no. 4, pp. 74-82, 2013, doi: 10.1109/MMM.2013.2248587.

[20] M. Otoom, N. Otoum, M. A. Alzubaidi, Y. Etoom, and R. Banihani, "An IoT-based framework for early identification and monitoring of COVID-19 cases," Biomedical Signal Processing and Control, vol. 62, 2020, Art. no. 102149, doi: 10.1016/j.bspc.2020.102149.

[21] W. D. S. Ting, L. Carin, V. Dzau, and T. Y. Wong, "Digital technology and COVID-19," Nature Medicine, vol. 26, pp. 459-461, 2020, doi: 10.1038/s41591-020-0824-5

[22] W. Colitti, K. Steenhaut, N. De Caro, B. Buta, and V. Dobrota, "Evaluation of constrained application protocol for wireless sensor networks," 2011 18th IEEE Workshop on Local \& Metropolitan Area Networks (LANMAN), Chapel Hill, NC, 2011, pp. 1-6, doi: 10.1109/LANMAN.2011.6076934.

[23] P. Thota and Y. Kim, "Implementation and comparison of M2M protocols for internet of things," 2016 4th Intl Conf on Applied Computing and Information Technology/3rd Intl Conf on Computational Science/Intelligence and Applied Informatics/1st Intl Conf on Big Data, Cloud Computing, Data Science \& Engineering (ACIT-CSII-BCD), Las Vegas, NV, 2016, pp. 43-48, doi: 10.1109/ACIT-CSII-BCD.2016.021.

[24] S. Katsikeas et al., "Lightweight \& secure industrial IoT communications via the MQ telemetry transport protocol," 2017 IEEE Symposium on Computers and Communications (ISCC), Heraklion, 2017, pp. 1193-1200, doi: 10.1109/ISCC.2017.8024687.

[25] S. R. Akbar, K. Amron, H. Mulya, and S. Hanifah, "Message queue telemetry transport protocols implementation for wireless sensor networks communication-A performance review," 2017 International Conference on Sustainable Information Engineering and Technology (SIET), Malang, 2017, pp. 107-112, doi: 10.1109/SIET.2017.8304118.

[26] H. Chaudhari, "Raspberry Pi Technology: A review," International Journal of Innovative and Emerging Research in Engineering, vol. 2, no. 3, pp. 83-87, 2015.

[27] "Raspberry Pi Foundation," Raspberrypi.org. [Online]. Available: https://www.raspberrypi.org/documentation/computers/gettingstarted.html (Accessed Jun. 20, 2021)

[28] "Eclipse Mosquitto ${ }^{\mathrm{TM}}$ Documentation," Mosquitto.org 2019. [Online]. Available: https://mosquitto.org/man/mosquitto-8.html (Accessed Jul. 13, 2021)

[29] M. Maclean, "Raspberry Pi: Measure, record, explore," Lean Publishing, pp. 59-124, 2016.

[30] Atmel Corporation, "8-bit AVR Microntroller with 4/8/16/32K bytes in-system programmable flash," ATmega 328P datasheet, 2019

[31] Maxim Integrated, 2014. "Pulse oximeter and heart-rate sensor IC for wearable health," datasheet, 19-7065, [Online]. Available: https://www.maximintegrated.com/en/products/sensors/MAX30100.html

[32] Melexis, MLX90614 family, 2019. "Single and dual zone infra-red thermometer in TO-39, datasheet," [Online]. Available: https://www.melexis.com/en/product/MLX90614/Digital-Plug-Play-Infrared-Thermometer-TO-Can

[33] Shanghai SIMCom Wireless Solutions Ltd, "SIM800L Hardware Design," version 1.0, 2013.

[34] Espressif Systems, "ESP8266EX Datasheet," Espressif Systems IOT Team, 2015.

[35] X. Wang et al., "Efficient measurement of round-trip link delays in software-defined networks," Journal of Network and Computer Applications, vol. 150, 2020, Art. no. 102468, doi: 10.1016/j.jnca.2019.102468

[36] "Wireshark frequently asked questions: What is wireshark," Wireshark.org. 2019. [Online]. Available: https://www.wireshark.org/faq.html\#_what_is_wireshark (Accessed Aug. 19, 2019). 